\title{
Generation of Mouse Basal Ganglia Diffusion Tractography Using 9.4T MRI
}

\author{
Jae-Hyuk Shim, Sang-Jin Im, A-Yoon Kim, Yong-Tae Kim, \\ Eun Bee Kim and Hyeon-Man Baek* \\ Department of Health Sciences and Technology, GAIHST, Gachon University, Incheon 21999, Korea
}

\begin{abstract}
Over the years, diffusion tractography has seen increasing use for comparing minute differences in connectivity of brain structures in neurodegenerative diseases and treatments. Studies on connectivity between basal ganglia has been a focal point for studying the effects of diseases such as Parkinson's and Alzheimer's, as well as the effects of treatments such as deep brain stimulation. Additionally, in previous studies, diffusion tractography was utilized in disease mouse models to identify white matter alterations, as well as biomarkers that occur in the progression of disease. However, despite the extensive use of mouse models to study model diseases, the structural connectivity of the mouse basal ganglia has been inadequately explored. In this study, we present the methodology of segmenting the basal ganglia of a mouse brain, then generating diffusion tractography between the segmented basal ganglia structures. Additionally, we compare the relative levels of connectivity of connecting fibers between each basal ganglia structure, as well as visualize the shapes of each connection. We believe that our results and future studies utilizing diffusion tractography will be beneficial for properly assessing some of the connectivity changes that are found in the basal ganglia of various mouse models.
\end{abstract}

Key words: Mouse, Basal Ganglia, MRI, Diffusion tractography

\section{INTRODUCTION}

Improvements to magnetic resonance imaging (MRI) have facilitated the process of imaging a patient's brain, making it capable to observe changes in a disease infected brain noninvasively (not requiring harmful radiation or insertion of any equipment into the brain). Studies have utilized various MRI techniques to measure certain qualities of the brain and detect biomarkers to identify diseases, such as neuromelanin in Parkinson's [1] and hippocampal volume loss in Alzheimer's [2]. However, there are current limita-

Received January 9, 2019, Revised March 5, 2019, Accepted March 25, 2019

* To whom correspondence should be addressed. TEL: 82-32-899-6678, FAX: 82-32-899-6677 e-mail:hmbaek98@gachon.ac.kr tions to human MRI, one of which includes the length of the time needed to take certain images for visualizing biomarkers with proper contrast and signal to noise ratio (SNR). Certain imaging techniques require longer scan time to be able to image certain deep brain structures like the substantia nigra, which can cause discomfort in the patient, as well as induce motions and artifacts. Additionally, it is very difficult to recreate certain environments due to the lack of control in the disease state of patients being imaged. For example, some patients with Parkinson's disease express non-motor symptoms rather than express the more commonly known tremors and rigidity [3], which makes studying early diagnosis of Parkinson's difficult. As such, mouse models are still being utilized for MRI studies. Using mouse models enables a lot more control over the experiment environment, with a large amount of disease models being available [4] as well as being able to image all states of the diseased brain. In addition, the issue with long imag- 
ing time becomes less of an issue with model brains, which allows for a clearer detection in biomarkers and consistent results.

Diffusion tensor imaging (DTI) has seen increased use in understanding neural networks between brain structures. Observing structural connections generated using DTI tractography between basal ganglia has been a focal point in studying progression of diseases like Parkinson's and Alzheimer's [5-7]. The basal ganglia are a group of interconnected subcortical structures that mediate various functions of the body, such as voluntary movements, learning and cognition [8]. Alterations to basal ganglia structural connections, observed by lowered intensities and factional anisotropy (FA) values of white matter connecting basal ganglia structures, have been associated with Parkinson's [9]. DTI has also been used to observe quantitative improvements in patients that underwent Parkinson's treatment such as deep brain stimulation. Previous studies regarding deep brain stimulation of basal ganglia structures like subthalamic nucleus have shown improvements in connectivity strength [10]. Additionally, deep brain stimulation of connections between the basal ganglia and cerebellum has shown visual improvements in reducing Parkinson's symptoms [11]. Despite the extensive studies on the potentials of utilizing DTI to study structural connectivity of the basal ganglia, the basal ganglia connectomes have not been yet established for the mouse, an important animal model used to test treatment for neuronal diseases.

DTI has been used historically to study various aspects of the mouse brain. In one study, DTI was used to observe the changes in the white matter of a developing mouse brain, as well as distinguishing cerebral structures using color coded anisotropy maps [12]. Another study utilized DTI to identify connections between the mouse amygdala and various targets, mainly to describe how visual and limbic systems were connected [13]. Additionally, DTI was used to study the deterioration of mouse tissues under various conditions. One study was able to characterize changes in structural connectivity of amyotrophic lateral sclerosis mouse spinal cord using DTI [14]. Studies were also able to detect DTI changes in the substantia nigra of 6-hydroxydopamine [15] and MPTP [16] Parkinson's mouse models. As such, utilizing DTI for studying the mouse basal ganglia has the potential for enhancing our understanding of mechanisms that influence or are influenced by the basal ganglia.

In this study, we present a comprehensive methodology in segmenting the basal ganglia of the mouse (e.g., globus pallidus external, globus pallidus internal, subthalamic nucleus, substantia nigra reticular, substantia nigra compact, caudoputamen), as well as generating a probabilistic tractography between each segmented structures using 9.4T MRI.

\section{MATERIALS AND METHODS}

\section{Mouse}

Animal experiments and procedures were carried out in compliance with the Lee Gil Ya Cancer and Diabetes Institutional Center of Animal Care and Use. A 8 week old C57BL/6N mouse was chosen for this experiment. Mouse was transcardially perfused and fixed with $4 \%$ paraformaldehyde and $0.1 \%$ Magnevist ${ }^{\circledR}$. After perfusion, the mouse was decapitated and had excess skin/muscle removed from the skull. The mouse brain with the remaining intact skulls were post-fixed in $0.1 \%$ Magnevist/phosphate buffer at $4^{\circ} \mathrm{C}$ for 4 days. Samples were removed from fixation solutions and tapped with a paper towel. Before imaging, brain, still intact with skulls were placed in a custom-made MRI compatible tube and were immersed in Fomblin [17].

The extracted brain was then imaged using a 9.4T Bruker Biospec horizontal bore, dedicated animal scanner (Bruker Biospin, Ettlingen, Germany). A four-channel receive-only phased array head coil was used for receiving. The T2-weighted images were acquired using a $3 \mathrm{D}$ turbo rare $\mathrm{T} 2$ sequence (echo time $(\mathrm{TE})=42.5$ $\mathrm{ms}$; repetition time $(\mathrm{TR})=3000 \mathrm{~ms}$; flip angle $=180^{\circ}$; field of view $($ FOV $)=1.2 \times 1.2 \times 1.2 \mathrm{~cm}^{3} ;$ matrix $=120 \times 120 \times 150$; bandwidth $=75$ $\mathrm{kHz}$; total imaging time $=52 \mathrm{~min}$ ) and the diffusion tensor images were acquired using 2D EPI diffusion tensor sequence (echo time $=33.63 \mathrm{~ms}$; repetition time $=12000 \mathrm{~ms}$; flip angle $=15^{\circ}$; field of view $=1.8 \times 1.8 \mathrm{~cm}^{2}$; matrix $=120 \times 120$; slice thickness $=150 \mu \mathrm{m}$; bandwidth $=300 \mathrm{kHz}$; b-value $=2000 \mathrm{~s} / \mathrm{mm}^{2}$; diffusion directions=30; total imaging time $=2 \mathrm{hr} 1 \mathrm{~min}$ ).

\section{Segmentation and tractography}

We referenced previous studies to model our experiment and figures for visualization $[18,19]$. To quantify the connections between each basal ganglia structure, we first segmented each basal ganglia structure from a diffusion image (shown in Fig. 1 in 3D structures, as well as their locations in the mouse brain). We then generated probabilistic tractography for each and between every segmented structure. For each segmented structure, we generated individual connectivity maps (shown in Fig. 2 as red fibers and blue structures overlaid on top of a T2 image), which represents the fibers that extend from the structure of interest to the rest of the brain. Additionally, utilizing waypoint connectivity mapping, we generated probabilistic tractography between two different regions (Fig. 3), which was done by generating random tractography that stems from one structure, and removing fibers that did not pass through the two chosen structures.

Segmentation of basal ganglia structures (e.g., globus pallidus external (GPe), globus pallidus internal (GPi), subthalamic nucleus 
(STN), substantia nigra reticular (SNr), substantia nigra compact $(\mathrm{SNc})$, caudoputamen $(\mathrm{CP})$ ) was done using Atlas Normalization Toolbox using elastiX (ANTX) [20-22], a MATLAB based toolbox, and FMRIB software library (FSL) [23]. The following steps were done using ANTX. The T2 and DTI DICOM files were converted into NIFTI files. The b0 image from the converted DTI NIFTI file were extracted for segmentation of structures. A modified version of SPM8, SPMMouse [24] was used to segment extracted b0 image into tissue compartment maps of grey and white matter, as well as the cerebrospinal fluid. Subsequently, by using ELASTIX [25], the extracted b0 image was normalized to Allen space using 12-parameter affine transformation and nonlinear image warping. The resulting transformation matrix generated from the normalization was used to transform Allen atlas labels (bilateral GPe, GPi, STN, SNr, SNc, CP) [26] back to native DTI space.

The following steps were done using FSL. The DTI image converted from ANTX were eddy corrected using FSL's eddycorrect [27]. Eddy correct is a tool for correcting current induced distortions and subject motion. Each DTI volume from the DTI image undergoes mutual information-based rigid-body registration to the $b=0$ image, reducing misalignment and distortion found in raw DTI image. After eddy correction, the diffusion tensors were fitted on eddy corrected DTI image using DTIFIT. After checking the results of DTIFIT for correct orientation of vector orientations, BEDPOSTX, Bayesian Estimation of Diffusion Parameters Obtained using Sampling Techniques [28], was run to model crossing fibers within each voxel of the DTI image (Fig. 4). BEDPOSTX operates by running Markov Chain Monte Carlo sampling to model distributions of diffusion parameters (fiber orientations) at each voxel. Number of fibers per voxel were set to three to account for multiple fiber orientations. Using the fiber orientation distributions applied by BEDPOSTX, probabilistic tractography was generated between the previously segmented structures (referred to as waypoints) using PROBTRACKX [29]. PROBTRACKX computes streamlines (in this case, 5000) through sampled fiber orientations of each voxel in a segmented region, then calculates the probability of the generated streamlines. The number of fiber counts generated through probabilistic tractography was used for statistical analysis.

For visual inspection and comparison of Allen Brain mouse neural tracer injection data and our diffusion connectivity data, injection (where neural tracers were injected, usually in structures) and projection (data describing the spread of neural tracer) data involving neural tracer experiments in segmented structures (GPe, GPi, STN, etc.) were obtained using Allen Mouse Brain Software Development Kit (http://alleninstitute.github.io/AllenSDK/connectivity.html). The template image, which is the Allen Mouse brain image space the injection and projection data are registered to, are first transformed into our mouse diffusion data space using ANTX coregistration. Using the generated matrix file describing the transformation of template space to diffusion space, the injection data was registered to our diffusion data space. After the registration, PROBTRACKX was run using the same settings used to run our native data and segmented masks, but using the newly registered injection data as seed mask. The resulting 3D fiber data was transformed back to Allen Mouse brain image space by using the inverse of the matrix generated to transform template image into our diffusion data space for tracer and diffusion tractography comparison.

\section{Statistics}

The number of fiber counts between pairs of segmented structures were analyzed. PROBTRACKX seeds fibers from the voxels of segmented regions, meaning that fibers generated using PROBTRACKX may vary depending on the volume of the segmented seed regions. To minimize this effect, the number of fiber counts
A

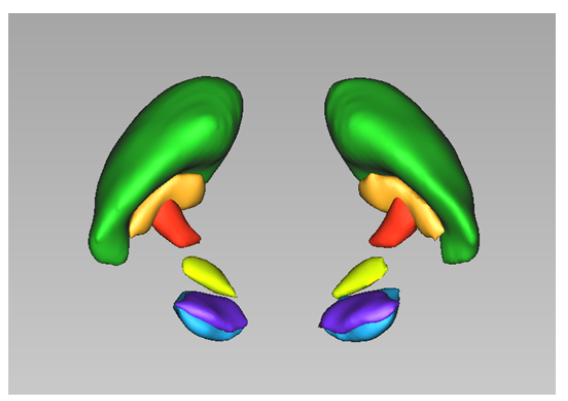

B

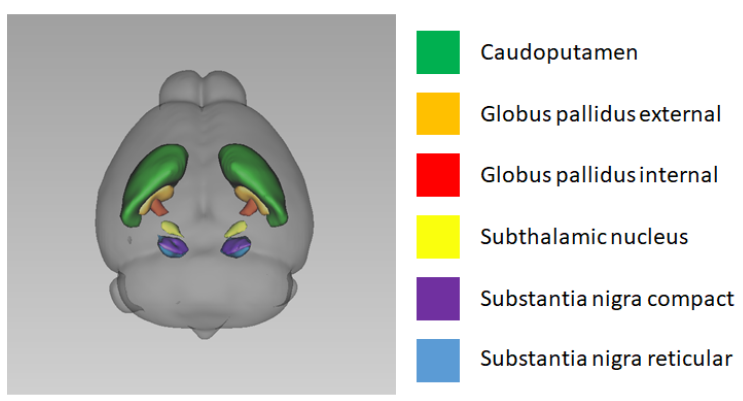

Fig. 1. 3D representations of segmented basal ganglia structures. (A) Segmented bilateral structures are color coded: green, caudoputamen; orange, globus pallidus external; red, globus pallidus internal; yellow, subthalamic nucleus; purple, substantia nigra compact; blue, substantia nigra reticular. (B) Segmented structures are displayed within a 3D transparent mouse brain rendering as a reference of their location within the mouse brain. 


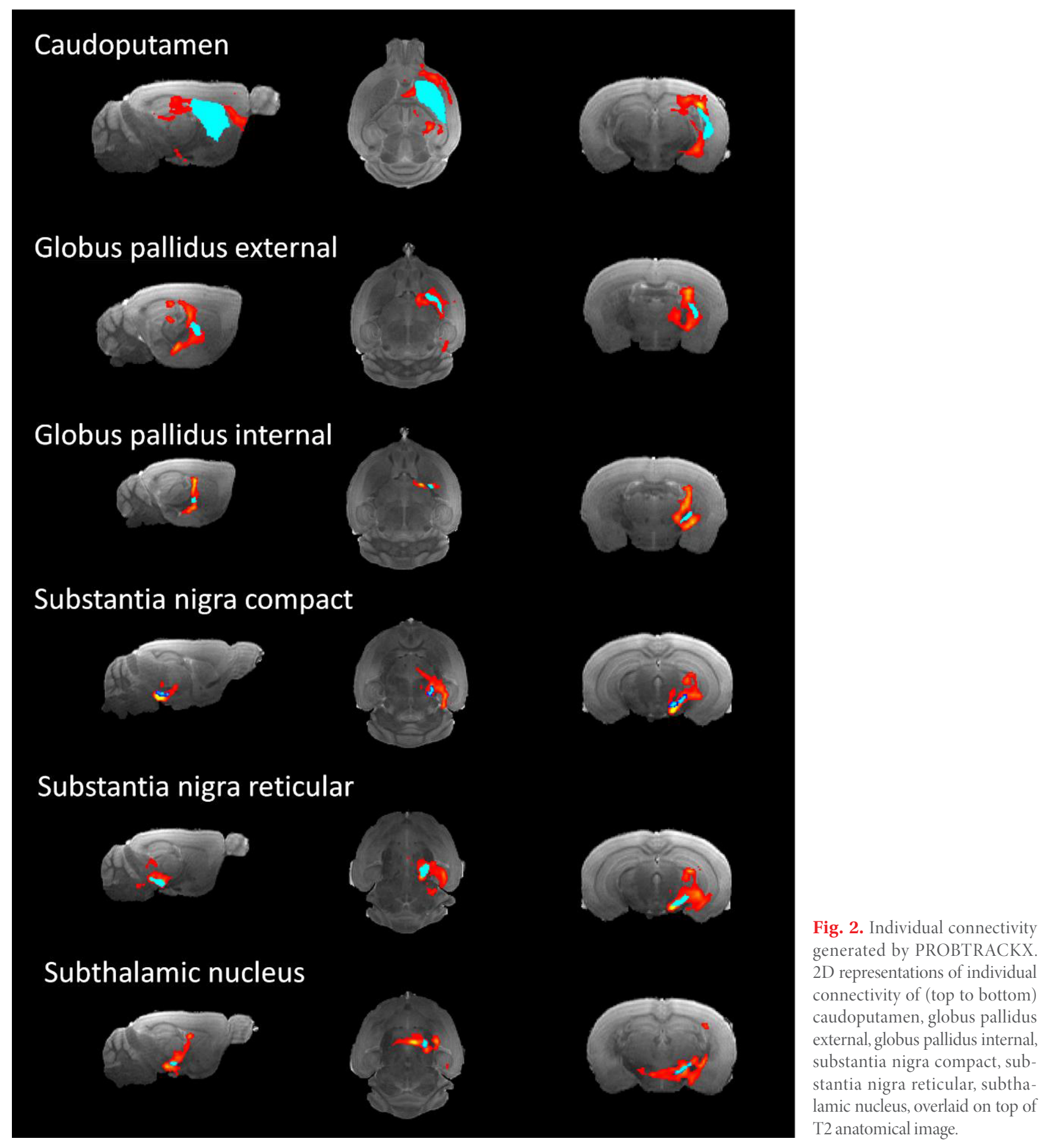

generated between two pairs (a seed and a target) were normalized by dividing the fiber counts by the seed volume. Normalized fiber counts of each pair were represented in percentage of total number of fibers connecting each pair to compare levels of connectivity between each basal ganglia structure. Additionally, the normalized fibers generated between left and left, left and right, right and left, right and right structures were organized into a connectivity matrix, represented in a $\log 10$ scale.

\section{RESULTS}

The percentage of connectivity between each segmented struc- 
A

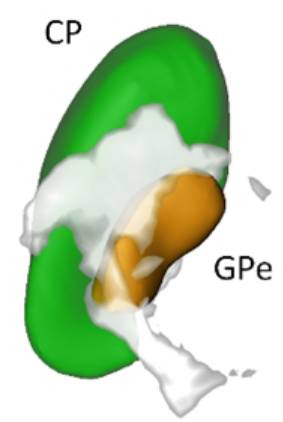

D

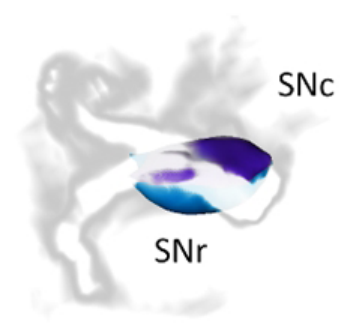

B

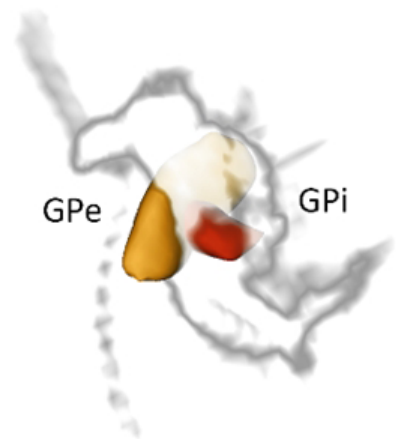

E

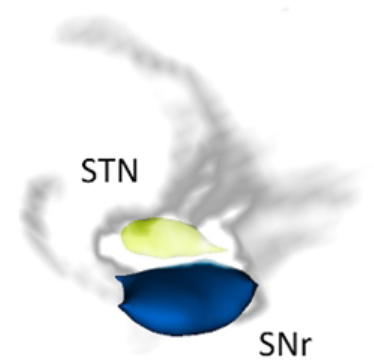

C

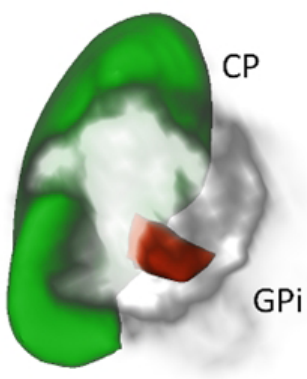

F

Fig. 3. Waypoint connectivity generated by PROBTRACKX. 3D representations of waypoint connectivity between structures with the highest relative levels of connectivity. (A) Waypoint connectivity between caudoputamen and globus pallidus external. (B) Waypoint connectivity between globus pallidus external and globus pallidus internal. (C) Waypoint connectivity between caudoputamen and globus pallidus internal. (D) Waypoint connectivity between substantia nigra reticular and substantia nigra compact. (E) Waypoint connectivity between subthalamic nucleus and substantia nigra reticular. (F) Waypoint connectivity between subthalamic nucleus and substantia nigra compact.

A

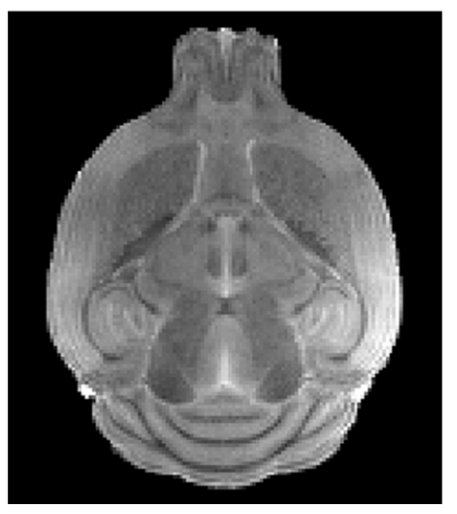

B

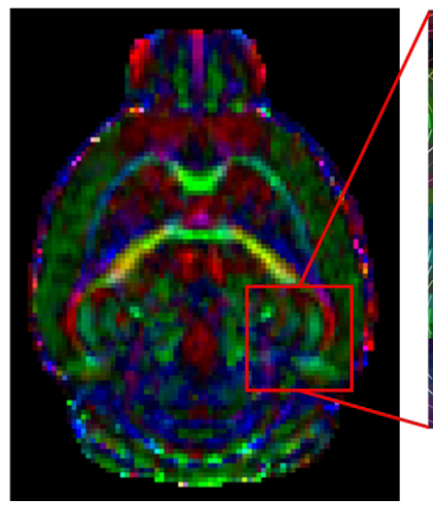

C

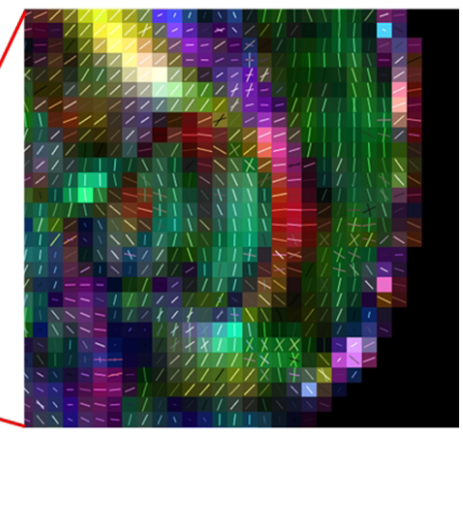

Fig. 4. Results from using BEDPOSTX to fit fiber orientations at each voxel. (A) T2 anatomic image of mouse brain used for diffusion tractography. (B) Colored fractional anisotrophy diffusion image used in this experiment. (C) Example of fiber orientations generated at each voxel using BEDPOSTX. Some voxels have multiple orientations (up to three). tures are shown in Table 1. The percentages describe the amount of normalized fibers that pass through the seed structure also passes through the target structure. Some percentages were lower than $100 \%$ due to the fact that not all fibers generated from a seed structure pass through the segmented structures and some percentages were higher than $100 \%$ due to the fact that most fibers that passed through a seed structure also passed through multiple target structures. Highest levels of connectivity were found mostly between structures that were closest to each other with $92.75 \%$ of fibers that pass through SNc connect to $\mathrm{SNr}$ and $87.36 \%$ of fibers that pass through GPe connect to CP. The lowest levels of connectivity were found between SNc and GPi at 0.04\% (with SNc as seed and GPi as target), and CP and SNc also at $0.04 \%$ (with CP as seed and SNc as target). The relative levels of direct connections (which were calculated by dividing each number of fibers by sum of the fibers generated between all structures) are shown in Table 2. Similar to 
Table 1, the connections were strongest between $\mathrm{SNr}$ and SNc at 92.7\% and between CP and GPi at 84.79\%. A visual representation of relative connectivity shown in Table 2 is shown in Fig. 5.

Waypoint connectivity using PROBTRACKX produced two sets of numbers, one for seed to target fibers and target to seed

Table 1. Percentages of fibers generated from seed structures that pass through target structures

\begin{tabular}{|c|c|c|c|c|c|c|}
\hline \multirow{3}{*}{$\begin{array}{c}\text { Target } \\
\text { struc- } \\
\text { tures }\end{array}$} & \multicolumn{6}{|c|}{ Seed structures } \\
\hline & GPe & GPi & STN & $\mathrm{SNr}$ & SNc & CP \\
\hline & $\%$ & $\%$ & $\%$ & $\%$ & $\%$ & $\%$ \\
\hline GPe & & 78.03 & 4.20 & 0.28 & 0.12 & 20.59 \\
\hline GPi & 35.35 & & 5.17 & 0.08 & 0.04 & 3.28 \\
\hline STN & 2.22 & 6.21 & & 11.40 & 6.67 & 0.25 \\
\hline $\mathrm{SNr}$ & 0.82 & 0.53 & 28.57 & & 92.04 & 0.12 \\
\hline $\mathrm{SNc}$ & 0.16 & 0.16 & 14.39 & 71.63 & & 0.04 \\
\hline $\mathrm{CP}$ & 87.36 & 48.79 & 5.51 & 0.58 & 0.35 & \\
\hline
\end{tabular}

Numbers represents the percentages of fibers generated from a seed structure that also reached a target structure. Each number was generated from the averages of left seed structure to left target structure and right seed structure to right target structure. Total percentages exceed 100\% for each seed structure due to fibers generated from seed structures connecting more than one target structure. Both target and seed structures are GPe, globus pallidus external; GPi, globus pallidus internal; STN, subthalamic nucleus; $\mathrm{SNr}$, substantia nigra reticular; $\mathrm{SNc}$, substantia nigra compact; $\mathrm{CP}$, caudoputamen. fibers. The number of fibers that are generated from probabilistic tractography are dependent on the seed region used for PROBTRACKX, which meant that the fibers generated between seed structure A and target structure B were different from the fibers

Table 2. Percentages of relative connectivity between a seed structure and target structures

\begin{tabular}{lcccccc}
\hline $\begin{array}{c}\text { Target } \\
\text { struc- } \\
\text { tures }\end{array}$ & GPe & GPi & STN & SNr & SNc & CP \\
\cline { 2 - 7 } GPe & - & $58.36 \%$ & $7.27 \%$ & $0.33 \%$ & $0.12 \%$ & $84.79 \%$ \\
GPi & $28.07 \%$ & - & $8.93 \%$ & $0.10 \%$ & $0.04 \%$ & $13.50 \%$ \\
STN & $1.76 \%$ & $4.64 \%$ & - & $13.57 \%$ & $6.73 \%$ & $1.04 \%$ \\
SNr & $0.65 \%$ & $0.40 \%$ & $49.40 \%$ & - & $92.75 \%$ & $0.49 \%$ \\
SNc & $0.13 \%$ & $0.12 \%$ & $24.88 \%$ & $85.31 \%$ & - & $0.18 \%$ \\
$\mathrm{CP}$ & $69.38 \%$ & $36.49 \%$ & $9.52 \%$ & $0.69 \%$ & $0.35 \%$ & - \\
\hline
\end{tabular}

Numbers represents the relative percentages of diffusion fibers generated between each seed structure and all other target structures. Each number was generated from the averages of left seed structure to left target structure and right seed structure to right target structure. The numbers were then divided by total fibers generated from a seed structure and other target structures, then represented in percentages. Both target and seed structures are GPe, globus pallidus external; GPi, globus pallidus internal; STN, subthalamic nucleus; $\mathrm{SNr}$, substantia nigra reticular; $\mathrm{SNc}$, substantia nigra compact; CP, caudoputamen.

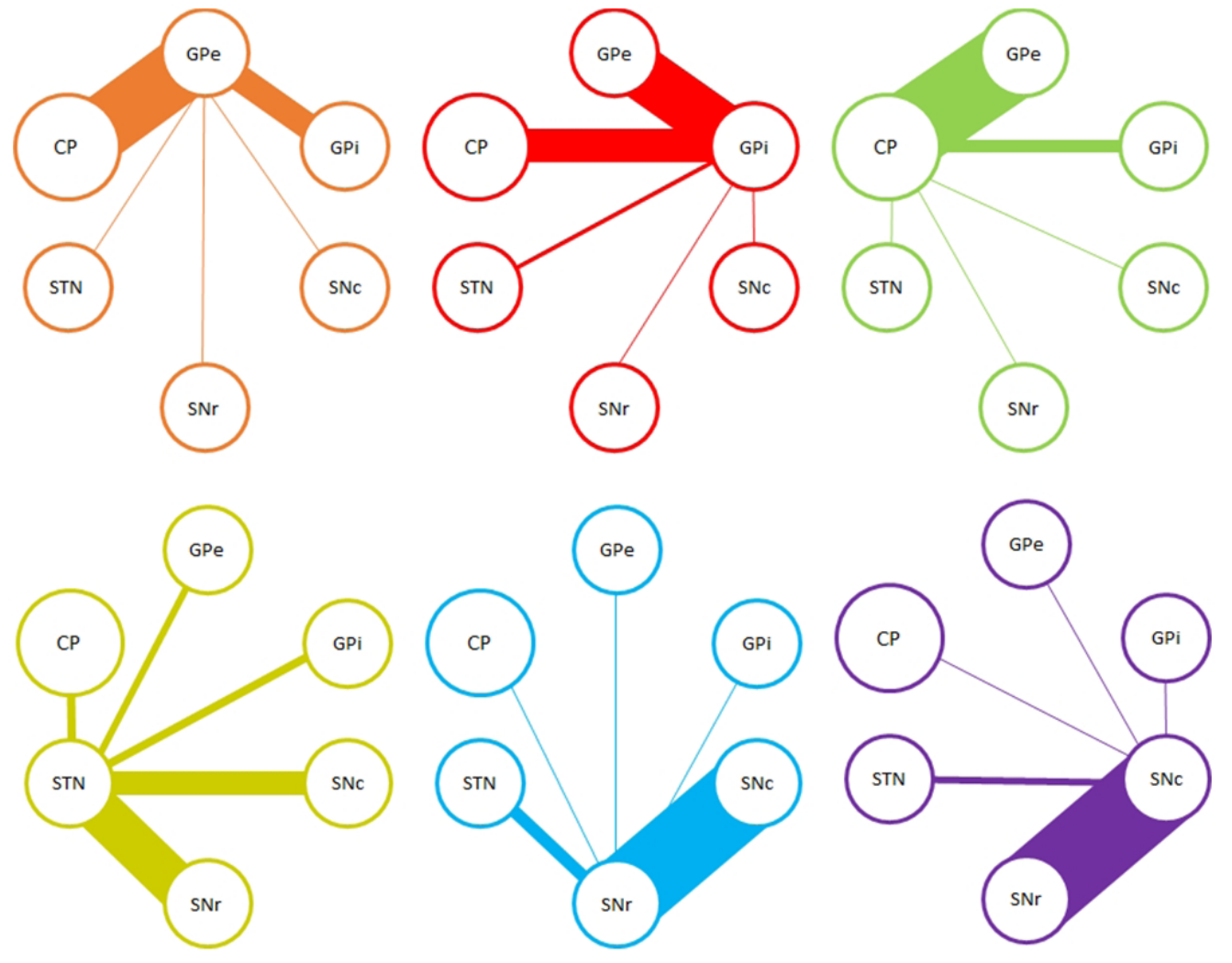

Fig. 5. Visual representation of Table 2. The thickness of lines connecting each structure, caudoputamen (CP), globus pallidus external (GPe), globus pallidus internal (GPi), substantia nigra compact $(\mathrm{SNc})$, substantia nigra reticular $(\mathrm{SNr})$, subthalamic nucleus (STN), represent relative levels of diffusion connectivity. 


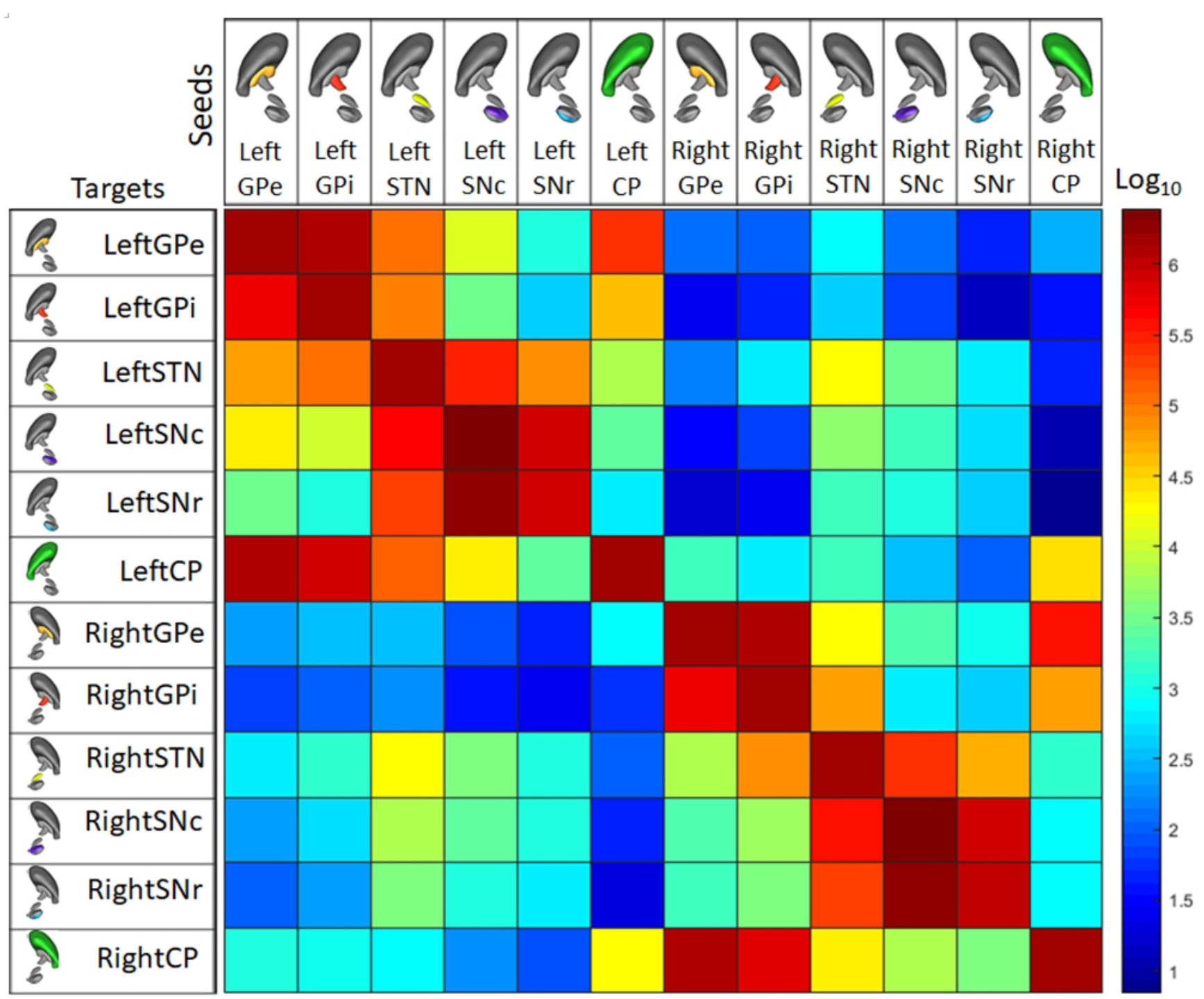

Fig. 6. Probabilistic tractography connectivity matrix of waypoint connectivity between left and right basal ganglia structures, displayed with a log 10 scale on the right. The seeds (top column) and targets (left row) are indicated by their name and colored 3D rendering. The diagonal elements of the matrix represent total number of fibers generated from each seed.

generated between seed structure B and target structure A. As a result, we quantified probabilistic tractography using left hemisphere structures as seeds and targets, right hemisphere structures as seeds and targets, then left hemisphere structures as seeds with right hemisphere structures as targets and vice versa. The resulting fibers were visualized as a $3 \mathrm{D}$ volumetric object. Using the data from probabilistic tractography, we generated a connectivity matrix representing the relative connectivity between the left and right seed structures and left and right target structures (Fig. 6).

To compare the results of our methodology with neuronal tracer data, we ran the DTI tractography pipeline using the 3D injection data of neuronal tracer as the seed structure. The injection structure (shown in blue in Fig. 7) was transformed into our native data space, then the results of our DTI tractography (shown in red in Fig. 7) was transformed into Allen Brain Atlas template space for direct comparison with projection data (shown in green in Fig. 7). Globus pallidus external (GPe) injection structure, projection structure and DTI tractography generated from GPe injection structure are overlaid on top of Allen Brain Atlas template in Fig. 7.

\section{DISCUSSION}

There has been a growing trend of studies that show significant changes in diffusion tractography in diseased individuals as well as in patients that have undergone treatment. Diffusion tractography utilizes diffusion tensor images to reconstruct white matter fiber 


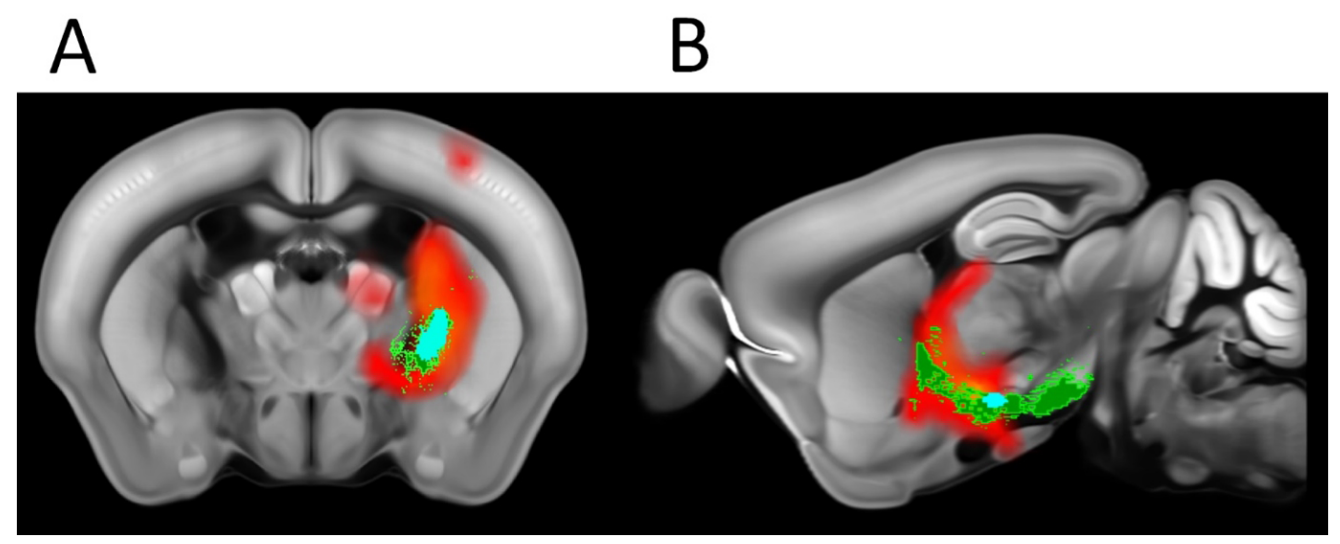

Fig. 7. Comparison between diffusion tractography and neuronal tracer projection. The neuronal injection site regions of interest (colored in light blue) were first registered to our native mouse brain diffusion image then used to generated diffusion tractography. Then the diffusion tractography fibers (colored in red-orange) generated from the injection ROI were registered back to ABA mouse template (obtained using ABA software development kit http://alleninstitute.github.io/AllenSDK/connectivity.html) and was compared with neuronal tracer projection data (colored in green). All injection, projection and diffusion tractography data were overlaid on top of ABA mouse template. (A) Sagittal view of the injection, projection, diffusion tractography, $\mathrm{ABA}$ template overlay. (B) Coronal view of the injection, projection, diffusion tractography, $\mathrm{ABA}$ template overlay.

bundles and pathways between brain structures. Various studies have used this tool to observe changes in white matter fibers of patients with Parkinson's disease [30] and Alzheimer's disease [6]. Additionally, there were studies that utilized diffusion tractography to observe increase in connectivity due to deep brain stimulation in Parkinson's patients [31]. As such, it is likely that diffusion tractography can be used to identify significant effects of preclinical treatment in animal models. While the use of probabilistic tractography has yet to be thoroughly explored in mice models, the ability to observe significant changes in the mouse brain without the need for invasive procedures should prove to be beneficial in improving the consistency of results.

In this study, we constructed probabilistic tractography between basal ganglia structures of a mouse. The relative levels of connectivity were often highest between structures that were closest to each other. Similarly to the Plantinga study [19], which used diffusion tractography to measure relative connectivity between the human basal ganglia, GPi was most strongly connected with GPe and SNc was most strongly connected with SNr (Fig. 3 and 5). There were couple of differences however, with Plantinga study reporting almost identical connectivity between $\mathrm{STN}, \mathrm{SNr}$ and STN, SNc, while our results showed stronger connections between STN and SNr than STN and SNc. It is possible that the differences in results are due to anatomical differences (regarding size and distance) between human brain structures and mouse brain structures [32].

There are benefits to exploring the structural connectivity between basal ganglia structures. The basal ganglia consist of interconnected subcortical structures responsible for mediating various motor and limbic functions. In diseases like Alzheimer's and Parkinson's, there are associated problems that arise in the basal ganglia, particularly in the disrupted flow of neurotransmitters between basal ganglia structures. Patients with Alzheimer's have shown depletion in choline acetyltransferase (precursor to acetylcholine) and dopamine [33], which are neurotransmitters responsible for motor control and reward systems [34, 35]. In patients with Parkinson's disease, it is widely accepted that dopamine producing cells are severely depleted (particularly in the substantia nigra), which disrupts the function of associative and sensorimotor striatum [36]. Using Parkinson's mouse models, which are produced by disrupting the dopaminergic pathway with toxins such as MPTP [4] or 6-OHDA [37], various studies showed issues within the basal ganglia. One study using Parkinsons mouse model showed beta oscillations in the cortico-basal ganglia loop, whose reduction seemed to correlate with alleviation of Parkinson's motor symptoms $[38,39]$. Other studies revealed alteration of glutamatergic neurotransmissions in the striatum and subthalamic nucleus of Parkinson's models [40]. Using Alzheimer's mouse models, studies were able to visualize the pathology of Alzheimer's disease in the basal ganglia [41] as well as demonstrate potential treatment in reducing Alzheimer's biomarkers which are usually found in the basal ganglia [42]. With numerous studies placing heavy emphasis on the basal ganglia regarding neurodegenerative diseases like Parkinson's and Alzheimer's, we believe our results and methodology should aid future experiments in identifying significant effects of pre-clinical treatments on neurodegenerative diseases.

While probabilistic diffusion tractography provides avenues for 
comparing structural connections between basal ganglia structures, results still remain questionable due to various factors such as lack of prior studies on probabilistic tractography between basal ganglia of the mouse, as well as imperfect data acquisition (which could lead to noise and artifacts interfering with connectome generation) [43]. Therefore, our generated tractography were directly compared with neuronal tracer data obtained from Allen Brain Atlas to test for the accuracy of using probabilistic diffusion tractography for mapping structural connectivity between basal ganglia structures (Fig. 7). However, similar to the Calabrese study [18], structural tractography generated using diffusion probabilistic tractography did not correspond well with neuronal tracer projection data. In Fig. 7, comparisons between DTI tractography and neural tracer projection shows differing travel directions, with DTI tractography expanding upwards while projection expands sideways. The Calabrese study also states that this is possibly due to the difference in nature of neuronal tracers, which travels unidirectional through individual axons, and diffusion tractography, which are generated multidirectionally (from fiber orientations) through multiple axons and in between synapses.

There are some limitations to consider for this study. First, only a single mouse was utilized for this study, limiting potential tests for validation and variability. We were unable to conduct tests to correct for individual variations of the mouse brain, which could have a significant effect on the segmentation of brain structures, as well as tractography generated between structures. Second, a postmortem mouse brain was utilized for this study. While perfusion fixing postmortem mouse brain can enhance the clarity of MRI images acquired [44], it is possible that the results can significantly differ when using a live, in vivo mouse. Additionally, there has been studies reporting that the elapsed time between death and perfusion fixation can adversely affect diffusion properties of mouse brain [45]. As such, it is possible that the results of our ex vivo segmentation and tractography can vary when compared with the results of in vivo mouse brain segmentation and tractography.

Diffusion tractography between the structures of the basal ganglia has yet to be studied using a mouse model. The present study quantified and visualized probabilistic tractography between basal ganglia structures of the mouse. With growing importance in using diffusion tractography to measure connectivity levels of the basal ganglia in patients with neurodiseases such as Parkinson's and Alzheimer's, we believe that our data will be useful in identifying alterations of the levels of connectivity in other mouse models, possibly with neurodiseases modelling Parkinson's and Alzheimer's. With additional studies on the use of diffusion tractography on mice and variety of disease mouse models, we believe that diffusion tractography can be an important avenue for studying the changes of structural connectivity that arises from neurodegenerative diseases.

\section{ACKNOWLEDGEMENTS}

This research was supported by Brain Research Program (NRF2017m3c7a1044367) and Korea Mouse Phenotyping Project (2014M3A9D5A01073886) through the National Research Foundation of Korea (NRF) funded by the Ministry of Science and ICT.

\section{REFERENCES}

1. Sulzer D, Cassidy C, Horga G, Kang UJ, Fahn S, Casella L, Pezzoli G, Langley J, Hu XP, Zucca FA, Isaias IU, Zecca L (2018) Neuromelanin detection by magnetic resonance imaging (MRI) and its promise as a biomarker for Parkinson's disease. NPJ Parkinsons Dis 4:11.

2. Schuff N, Woerner N, Boreta L, Kornfield T, Shaw LM, Trojanowski JQ, Thompson PM, Jack CR Jr, Weiner MW; Alzheimer's Disease Neuroimaging Initiative (2009) MRI of hippocampal volume loss in early Alzheimer's disease in relation to ApoE genotype and biomarkers. Brain 132:1067-1077.

3. Sauerbier A, Jenner P, Todorova A, Chaudhuri KR (2016) Non motor subtypes and Parkinson's disease. Parkinsonism Relat Disord 22 Suppl 1:S41-S46.

4. Jackson-Lewis V, Przedborski S (2007) Protocol for the MPTP mouse model of Parkinson's disease. Nat Protoc 2:141-151.

5. Cochrane CJ, Ebmeier KP (2013) Diffusion tensor imaging in parkinsonian syndromes: a systematic review and metaanalysis. Neurology 80:857-864.

6. Lo CY, Wang PN, Chou KH, Wang J, He Y, Lin CP (2010) Diffusion tensor tractography reveals abnormal topological organization in structural cortical networks in Alzheimer's disease. J Neurosci 30:16876-16885.

7. Doan NT, Engvig A, Persson K, Alnæs D, Kaufmann T, Rokicki J, Córdova-Palomera A, Moberget T, Brækhus A, Barca ML, Engedal K, Andreassen OA, Selbæk G, Westlye LT (2017) Dissociable diffusion MRI patterns of white matter microstructure and connectivity in Alzheimer's disease spectrum. Sci Rep 7:45131.

8. Stocco A, Lebiere C, Anderson JR (2010) Conditional routing of information to the cortex: a model of the basal ganglias role in cognitive coordination. Psychol Rev 117:541-574.

9. Zhang Y, Wu IW, Buckley S, Coffey CS, Foster E, Mendick S, Seibyl J, Schuff N (2015) Diffusion tensor imaging of the nigrostriatal fibers in Parkinson’s disease. Mov Disord 30:12291236. 
10. Vanegas-Arroyave N, Lauro PM, Huang L, Hallett M, Horovitz SG, Zaghloul KA, Lungu C (2016) Tractography patterns of subthalamic nucleus deep brain stimulation. Brain 139:1200-1210.

11. Sweet JA, Walter BL, Gunalan K, Chaturvedi A, McIntyre CC, Miller JP (2014) Fiber tractography of the axonal pathways linking the basal ganglia and cerebellum in Parkinson disease: implications for targeting in deep brain stimulation. J Neurosurg 120:988-996.

12. Mori S, Itoh R, Zhang J, Kaufmann WE, van Zijl PC, Solaiyappan M, Yarowsky P (2001) Diffusion tensor imaging of the developing mouse brain. Magn Reson Med 46:18-23.

13. Harsan LA, Paul D, Schnell S, Kreher BW, Hennig J, Staiger JF, von Elverfeldt D (2010) In vivo diffusion tensor magnetic resonance imaging and fiber tracking of the mouse brain. NMR Biomed 23:884-896.

14. Gatto RG, Amin MY, Deyoung D, Hey M, Mareci TH, Magin RL (2018) Ultra-high field diffusion MRI reveals early axonal pathology in spinal cord of ALS mice. Transl Neurodegener 7:20.

15. Van Camp N, Blockx I, Verhoye M, Casteels C, Coun F, Leemans A, Sijbers J, Baekelandt V, Van Laere K, Van der Linden A (2009) Diffusion tensor imaging in a rat model of Parkinson's disease after lesioning of the nigrostriatal tract. NMR Biomed 22:697-706.

16. Boska MD, Hasan KM, Kibuule D, Banerjee R, McIntyre E, Nelson JA, Hahn T, Gendelman HE, Mosley RL (2007) Quantitative diffusion tensor imaging detects dopaminergic neuronal degeneration in a murine model of Parkinson's disease. Neurobiol Dis 26:590-596.

17. Ullmann JF, Watson C, Janke AL, Kurniawan ND, Paxinos G, Reutens DC (2014) An MRI atlas of the mouse basal ganglia. Brain Struct Funct 219:1343-1353.

18. Calabrese E, Badea A, Cofer G, Qi Y, Johnson GA (2015) A diffusion MRI tractography connectome of the mouse brain and comparison with neuronal tracer data. Cereb Cortex 25:4628-4637.

19. Plantinga BR, Roebroeck A, Kemper VG, Uludağ K, Melse M, Mai J, Kuijf ML, Herrler A, Jahanshahi A, Ter Haar Romeny BM, Temel Y (2016) Ultra-high field MRI post mortem structural connectivity of the human subthalamic nucleus, substantia nigra, and globus pallidus. Front Neuroanat 10:66.

20. Koch S, Mueller S, Foddis M, Bienert T, von Elverfeldt D, Knab F, Farr TD, Bernard R, Dopatka M, Rex A, Dirnagl U, Harms C, Boehm-Sturm P (2019) Atlas registration for edema-corrected MRI lesion volume in mouse stroke models. J Cereb Blood Flow Metab 39:313-323.
21. Hübner NS, Mechling AE, Lee HL, Reisert M, Bienert T, Hennig J, von Elverfeldt D, Harsan LA (2017) The connectomics of brain demyelination: functional and structural patterns in the cuprizone mouse model. Neuroimage 146:1-18.

22. Lein ES, Hawrylycz MJ, Ao N, Ayres M, Bensinger A, Bernard A, Boe AF, Boguski MS, Brockway KS, Byrnes EJ, Chen L, Chen L, Chen TM, Chin MC, Chong J, Crook BE, Czaplinska A, Dang CN, Datta S, Dee NR, Desaki AL, Desta T, Diep E, Dolbeare TA, Donelan MJ, Dong HW, Dougherty JG, Duncan BJ, Ebbert AJ, Eichele G, Estin LK, Faber C, Facer BA, Fields R, Fischer SR, Fliss TP, Frensley C, Gates SN, Glattfelder KJ, Halverson KR, Hart MR, Hohmann JG, Howell MP, Jeung DP, Johnson RA, Karr PT, Kawal R, Kidney JM, Knapik RH, Kuan CL, Lake JH, Laramee AR, Larsen KD, Lau C, Lemon TA, Liang AJ, Liu Y, Luong LT, Michaels J, Morgan JJ, Morgan RJ, Mortrud MT, Mosqueda NF, Ng LL, Ng R, Orta GJ, Overly CC, Pak TH, Parry SE, Pathak SD, Pearson OC, Puchalski RB, Riley ZL, Rockett HR, Rowland SA, Royall JJ, Ruiz MJ, Sarno NR, Schaffnit K, Shapovalova NV, Sivisay T, Slaughterbeck CR, Smith SC, Smith KA, Smith BI, Sodt AJ, Stewart NN, Stumpf KR, Sunkin SM, Sutram M, Tam A, Teemer CD, Thaller C, Thompson CL, Varnam LR, Visel A, Whitlock RM, Wohnoutka PE, Wolkey CK, Wong VY, Wood M, Yaylaoglu MB, Young RC, Youngstrom BL, Yuan XF, Zhang B, Zwingman TA, Jones AR (2007) Genome-wide atlas of gene expression in the adult mouse brain. Nature 445:168-176.

23. Jenkinson M, Beckmann CF, Behrens TE, Woolrich MW, Smith SM (2012) Fsl. Neuroimage 62:782-790.

24. Sawiak SJ, Wood NI, Williams GB, Morton AJ, Carpenter TA (2009) SPMMouse: a new toolbox for SPM in the animal brain. In: Proceedings of 17th Annual ISMRM Scientific Meeting and Exhibition 2009; 2009 Apr 18-24; Honolulu, Hawaii.

25. Klein S, Staring M, Murphy K, Viergever MA, Pluim JP (2010) elastix: a toolbox for intensity-based medical image registration. IEEE Trans Med Imaging 29:196-205.

26. Oh SW, Harris JA, Ng L, Winslow B, Cain N, Mihalas S, Wang Q, Lau C, Kuan L, Henry AM, Mortrud MT, Ouellette B, Nguyen TN, Sorensen SA, Slaughterbeck CR, Wakeman W, Li Y, Feng D, Ho A, Nicholas E, Hirokawa KE, Bohn P, Joines KM, Peng H, Hawrylycz MJ, Phillips JW, Hohmann JG, Wohnoutka P, Gerfen CR, Koch C, Bernard A, Dang C, Jones AR, Zeng H (2014) A mesoscale connectome of the mouse brain. Nature 508:207-214.

27. Andersson JL, Sotiropoulos SN (2016) An integrated approach to correction for off-resonance effects and subject movement in diffusion MR imaging. Neuroimage 125:1063- 
1078.

28. Behrens TE, Woolrich MW, Jenkinson M, Johansen-Berg H, Nunes RG, Clare S, Matthews PM, Brady JM, Smith SM (2003) Characterization and propagation of uncertainty in diffusion-weighted MR imaging. Magn Reson Med 50:10771088.

29. Behrens TE, Berg HJ, Jbabdi S, Rushworth MF, Woolrich MW (2007) Probabilistic diffusion tractography with multiple fibre orientations: What can we gain? Neuroimage 34:144-155.

30. Surova Y, Szczepankiewicz F, Lätt J, Nilsson M, Eriksson B, Leemans A, Hansson O, van Westen D, Nilsson C (2013) Assessment of global and regional diffusion changes along white matter tracts in parkinsonian disorders by MR tractography. PLoS One 8:e66022.

31. Sweet JA, Walter BL, Gunalan K, Chaturvedi A, McIntyre CC, Miller JP (2014) Fiber tractography of the axonal pathways linking the basal ganglia and cerebellum in Parkinson disease: implications for targeting in deep brain stimulation. J Neurosurg 120:988-996.

32. Hardman CD, Henderson JM, Finkelstein DI, Horne MK, Paxinos G, Halliday GM (2002) Comparison of the basal ganglia in rats, marmosets, macaques, baboons, and humans: volume and neuronal number for the output, internal relay, and striatal modulating nuclei. J Comp Neurol 445:238-255.

33. Langlais PJ, Thal L, Hansen L, Galasko D, Alford M, Masliah E (1993) Neurotransmitters in basal ganglia and cortex of Alzheimer's disease with and without Lewy bodies. Neurology 43:1927-1934.

34. Salamone JD (1992) Complex motor and sensorimotor functions of striatal and accumbens dopamine: involvement in instrumental behavior processes. Psychopharmacology (Berl) 107:160-174.

35. Weiner DM, Levey AI, Brann MR (1990) Expression of muscarinic acetylcholine and dopamine receptor mRNAs in rat basal ganglia. Proc Natl Acad Sci U S A 87:7050-7054.

36. Ashby FG, Turner BO, Horvitz JC (2010) Cortical and basal ganglia contributions to habit learning and automaticity.
Trends Cogn Sci 14:208-215.

37. Schober A (2004) Classic toxin-induced animal models of Parkinson's disease: 6-OHDA and MPTP. Cell Tissue Res 318:215-224.

38. Kühn AA, Tsui A, Aziz T, Ray N, Brücke C, Kupsch A, Schneider GH, Brown P (2009) Pathological synchronisation in the subthalamic nucleus of patients with Parkinson's disease relates to both bradykinesia and rigidity. Exp Neurol 215:380387.

39. Beck MH, Haumesser JK, Kühn J, Altschüler J, Kühn AA, van Riesen C (2016) Short- and long-term dopamine depletion causes enhanced beta oscillations in the cortico-basal ganglia loop of parkinsonian rats. Exp Neurol 286:124-136.

40. Villalba RM, Mathai A, Smith Y (2015) Morphological changes of glutamatergic synapses in animal models of Parkinson's disease. Front Neuroanat 9:117.

41. Maruyama M, Shimada H, Suhara T, Shinotoh H, Ji B, Maeda J, Zhang MR, Trojanowski JQ, Lee VM, Ono M, Masamoto K, Takano H, Sahara N, Iwata N, Okamura N, Furumoto S, Kudo Y, Chang Q, Saido TC, Takashima A, Lewis J, Jang MK, Aoki I, Ito H, Higuchi M (2013) Imaging of tau pathology in a tauopathy mouse model and in Alzheimer patients compared to normal controls. Neuron 79:1094-1108.

42. Darvesh S, Reid GA (2016) Reduced fibrillar $\beta$-amyloid in subcortical structures in a butyrylcholinesterase-knockout Alzheimer disease mouse model. Chem Biol Interact 259:307-312.

43. Thomas C, Ye FQ, Irfanoglu MO, Modi P, Saleem KS, Leopold DA, Pierpaoli C (2014) Anatomical accuracy of brain connections derived from diffusion MRI tractography is inherently limited. Proc Natl Acad Sci U S A 111:16574-16579.

44. Johnson GA, Cofer GP, Gewalt SL, Hedlund LW (2002) Morphologic phenotyping with MR microscopy: the visible mouse. Radiology 222:789-793.

45. D'Arceuil H, de Crespigny A (2007) The effects of brain tissue decomposition on diffusion tensor imaging and tractography. Neuroimage 36:64-68. 